\title{
PESQUISA DO LINFONODO SENTINELA EM CÂNCER GÁSTRICO COM O CORANTE AZUL PATENTE
}

\author{
SENTINEL LYMPH NODE MAPPING IN GASTRIC CANCER USING PATENT BLUE DYE
}

\author{
Márcio Fernando Boff, ACBC-RS'; Carlos Cauduru Schirmer TCBC-RS²;
} Maria Isabel Albano Edelweiss ${ }^{3}$; Luise Meurer $^{4}$

\begin{abstract}
RESUMO: Objetivo: Avaliar a factibilidade e os resultados de uma técnica pouco invasiva para a pesquisa do linfonodo sentinela (LFNsn) com o uso do corante vital azul patente. Método: A pesquisa do LFNsn foi realizada em 12 pacientes portadores de adenocarcinoma gástrico que não apresentavam linfonodos suspeitos de metastases durante o estadiamento clínico pré-operatório. Injetou-se nos quatro quadrantes do tumor, $0,5 \mathrm{ml}$ de corante vital azul patente. Os linfonodos que se coraram de azul foram classificados como LFNsn e foram obtidos após a gastrectomia com linfadenectomia D2. Utilizou-se a coloração HE para avaliação anatomopatológica dos LFNsn, e nos casos com LFNsn negativo para HE, foi realizada imuno-histoquímica com pan-citoqueratinas AE1/AE3. Resultados: Dos 12 pacientes, oito eram mulheres, com média de idade de 64,5 (48-87) anos. Identificaram-se em média 3,25 (2-6) LFNsn por paciente. A factibilidade da técnica foi de 100\%. A acurácia do método foi de $91,6 \%$. Em 11 de 12 casos foi possível predizer as características linfonodais regionais pela concordância da análise anatomopatológica entre os LFNsn e não sentinelas(LFNñsn). Conclusão: A pesquisa do linfonodo sentinela em câncer gástrico é factível com o uso da técnica do corante azul patente. $\mathrm{O}$ método mostrou ser promissor como técnica minimamente invasiva para estadiar tumores gástricos, nesta casuística inicial (Rev. Col. Bras. Cir. 2007; 34(6): 367-373).
\end{abstract}

Descritores: Neoplasias gástricas; Metástase linfática; Estadiamento de neoplasias; Biópsia de linfonodo sentinela; Cirurgia assistida por computador.

\section{INTRODUÇÃO}

O linfonodo sentinela em câncer gástrico está sendo cada vez mais estudado. A partir do final dos anos 90, encontramos muitos relatos que evidenciaram este fato ${ }^{1-14}$.

Os três métodos para se realizar a pesquisa do linfonodo sentinela em câncer gástrico utilizam: o corante isolado, o radiotraçador isolado e a combinação de ambos ${ }^{11,13}$. A principal indicação para pesquisa do linfonodo sentinela em câncer gástrico parece ser em tumores precoces T1, devido às baixas probabilidades de metástases para linfonodos ${ }^{5,15-18}$. No entanto, nenhuma publicação relata restrição do seu uso em tumores avançados (T2,T3), apenas deve-se ter idéia de que quanto maior o grau de invasão tumoral maiores as chances de casos com LFNsn falso negativo ${ }^{12}$

Os dois tumores nos quais é realizada a pesquisa do LFNsn, com resultados satisfatórios, são o melanoma e o câncer de mama ${ }^{19-21}$. A técnica da pesquisa do linfonodo sentinela caracteriza-se principalmente em poder predizer com um alto percentual de acerto quais são as características dos demais linfonodos regionais, sendo importantíssima para estadiar estes tumores ${ }^{22-24}$

Para avaliar a factibilidade e os resultados de uma técnica pouco invasiva para a pesquisa do linfonodo senti- nela (LFNsn) com o uso do corante vital azul patente, decidimos realizar um estudo clínico em pacientes portadores de câncer gástrico em nosso meio.

\section{MÉTODO}

O estudo foi realizado em 12 pacientes portadores de adenocarcinoma gástrico no Hospital de Clínicas de Porto Alegre e em casos privados do autor, no período de junho de 2002 a dezembro de 2004.

Os critérios de inclusão foram os seguintes: adenocarcinoma gástrico localizado no fundo, corpo ou antro, lesões T1,T2 e T3, linfonodos regionais sem evidência de metástases no estadiamento clínico, tumor único e ressecável.

Os critérios de exclusão foram os seguintes: operações gástricas prévias, radioterapia ou quimioterapia prévias, doença metastática regional ou sistêmica, linfonodos regionais maiores que dois centímetros ou fusionados, operação paliativa, tumor irressecável, neoplasia sincrônica gástrica, lesão em trajetos linfáticos, tumores da junção esôfago-gástrica, alergia ao corante, e tumores T4.

O método escolhido para a localização do linfonodo sentinela foi o uso do corante azul patente V $(2,5 \%$, Guerbet-RJ ${ }^{\circledR}$ ). A técnica cirúrgica utilizada foi laparotomia

1. Cirurgião Geral e Oncológico; Mestre em Cirurgia pela UFRGS

2. Cirurgião Geral; Professor de Cirurgia na UFRGS; Mestre e Doutor pela UFRGS

3. Patologista; Mestre pela UFRGS; Doutora pela USP; Orientadora de Pós-Graduação na UFRGS.

4. Patologista; Mestre e Doutora pela UFRGS.

Recebido em 09/04/2007

Aceito para publicação em 12/06/2007

Conflito de interesses: nenhum

Fonte de financiamento: nenhuma

Trabalho Realizado no Hospital de Clínicas de Porto Alegre. 
mediana supra-umbilical, seguida de revisão da cavidade abdominal e liberação de aderências não gástricas, quando necessário. Nos pacientes em que o tumor era considerado de pequenas dimensões e com possibilidade de não localizá-lo pelo método da palpação, solicitava-se, no pré-operatório, marcação endoscópica com clips metálicos para facilitar a identificação topográfica no trans-operatório. Localizado o tumor, injetava-se na sub-serosa o corante em quatro quadrantes da lesão no volume de $0,5 \mathrm{ml}$ por quadrante através de uma seringa de $3 \mathrm{ml}$ com agulha de 24 gauge, diminuindo assim, o extravasamento de corante na cavidade. O grande epíploon era liberado em sua porção avascular na borda do cólon transverso para permitir o acesso aos LFNsn posteriores ao estômago. Os LFNsn foram aqueles que se coraram de azul após cinco minutos da injeção do corante ${ }^{5,6,8,9}$. Estes linfonodos foram dissecados isoladamente antes de iniciar a ressecção gástrica e foram enviados separados ao Serviço de Patologia da instituição para serem analisados.

A gastrectomia era realizada de acordo com a localização tumoral e a linfadenectomia recomendada era a D2. O sistema de estadiamento foi o TNM-2003 ${ }^{25}$.

A rotina de avaliação dos linfonodos não sentinelas (LFNñsn) foi feita com a inclusão total das duas metades do linfonodo (LFN) que foram seccionados com um corte longitudinal em seu maior eixo. Os LFNsn foram, assim como os demais linfonodos, inclusos totalmente em parafina em suas duas metades. O estudo inicial dos LFNsn e LFNñsn ocorreu através análise de duas secções de quatro micrômetros e coloração com hematoxilina e eosina (HE). Nos que os LFNsn não apresentavam metástases ao exame com HE, eram realizados cortes seriados e associada a técnica imuno-histoquímica, com o uso de pan-citoqueratinas. As pan-citoqueratinas AE1/ AE3, da Dako®, foram utilizadas como marcadores de membrana celular.

O tipo tumoral foi descrito pela classificação de Láuren , o grau histológico foi definido pelo patologista variando de : Grau I - bem diferenciado,Grau II - moderadamente diferenciado, Grau III - pouco diferenciado. A classificação macroscópica do tumor foi feita segundo o parâmetro da classificação japonesa para os tumores gástricos ${ }^{26}$.
A técnica de processamento dos linfonodos sentinelas foi a seguinte: desbaste do bloco de parafina, seguida da realização de um corte para HE (usual) e uma lâmina para imunohistoquímica (IHQ). A partir destas lâminas iniciais foram realizados cortes escalonados, com 20 micrômetros de intervalo, dois níveis em cada lâmina, corados pela HE e, no corte imediatamente seguinte, para IHQ até o esgotamento do material no bloco.

A nomenclatura utilizada para qualificar as metástases linfonodais encontradas foi: - Macrometástase (metástases maiores que $2 \mathrm{~mm}$ ) / -Micrometástase(metástases entre 0,2 e 2 $\mathrm{mm}$ ) / - "Cluster" (Grupamento de células menores que 0,2 $\mathrm{mm})$ / - Células neoplásicas isoladas e células na luz de vasos.

Quanto à análise imuno-histoquímica os LFNsn foram processadas conforme a rotina do Serviço de Patologia do Hospital de Clínicas de Porto Alegre e do Centro de Pesquisa do Hospital de Clínicas de Porto Alegre.

As reações imuno-histoquímicas foram realizadas no laboratório de Patologia do Centro de Pesquisa do Hospital de Clínicas de Porto Alegre. Foi utilizado o anticorpo monoclonal anti-citoqueratina AE1/AE3 (DakoCytomation, Carpinteria, Califórnia, USA) diluído em tampão PBS à razão de 1:100. Para a determinação da positividade nestes tecidos foi utilizado o método ABS (complexo streptavidina-biotinaperoxidase; LSAB + System HRP, DakoCytomation, Carpinteria, Califórnia, USA).

As reações foram realizadas com controle positivo (adenocarcinoma intestinal) e controle negativo (omitindo-se o anticorpo primário).

O projeto de pesquisa e o termo de consentimento informado foram aprovados pelo Comitê de Ética em Pesquisa do Hospital das Clínicas de Porto Alegre.

\section{RESULTADOS}

Dos 12 pacientes, oito eram mulheres e quatro homens , com média de idade de 64,5 anos (48-87). O tamanho médio tumoral foi de $3,11 \mathrm{~cm}(1,3-7,0)$.

As características dos pacientes estão descritas na Tabela 1

Tabela 1 - Características dos pacientes, localização e tamanho dos tumores.

\begin{tabular}{|c|c|c|c|c|c|}
\hline Pacientes & Sexo & Idade & $\begin{array}{c}\text { Localização } \\
\text { dotumor }\end{array}$ & $\begin{array}{l}\text { Localização } \\
\text { circunferêncial }\end{array}$ & $\begin{array}{c}\text { Tamanho } \\
\text { tumor }(\mathbf{c m})\end{array}$ \\
\hline$\# 1$ & feminino & 76 & antro & pequena curvatura & 3 \\
\hline$\# 2$ & feminino & 58 & corpo & parede anterior & 2,5 \\
\hline$\# 3$ & feminino & 54 & corpo & parede posterior & 7 \\
\hline$\# 4$ & feminino & 58 & antro & pequena curvatura & 3 \\
\hline$\# 5$ & masculino & 74 & antro & pequena curvatura & 6 \\
\hline \#6 & feminino & 87 & antro & pequena curvatura & 3 \\
\hline$\# 7$ & feminino & 78 & antro & grande curvatura & 2,5 \\
\hline$\# 8$ & masculino & 68 & antro & parede posterior & 2,5 \\
\hline \#9 & masculino & 66 & antro & parede anterior & 2,5 \\
\hline$\# 10$ & feminino & 52 & antro & pequena curvatura & 1,8 \\
\hline \#11 & feminina & 48 & fundo & parede anterior & 1,3 \\
\hline \#12 & masculino & 56 & antro & pequena curvatura & 2,3 \\
\hline
\end{tabular}


As características dos tumores e o tipo de gastrectomia estão citadas na Tabela 2.

Todos pacientes apresentavam estadiamento clínico pré-operatório negativo para metástases linfonodais e após estudo anatomopatológico foram encontrados sete casos com presença de metástases em linfonodos. O número médio de linfonodos dissecados foi de 19,6 (10-27) por paciente, de um total de 236 linfonodos avaliados (Tabela 3 ).

Foram identificados em média 3,25 (2-6) linfonodos sentinelas por paciente. A localização dos linfonodos sentinelas em relação às estações linfonodais pela classificação japonesa foi de, pelo menos um LFNsn em estação perigástricas N1 e em oito pacientes os linfonodos sentinelas localizaramse em estações extra-perigástricas N2. Em cinco pacientes foram encontrados LFNsn com presença de metástases. Um paciente (\#6) foi considerado falso negativo pois os LFNsn não apresentavam metástases enquanto os LFNñsn eram metastáticos (Figura 1).

Foram encontrados seis pacientes com ausência de metástases em LFNsn. No paciente \#12 foi encontrado um LFNsn positivo para micrometástases detectado pela IHQ e neste mesmo paciente foi encontrado outro LFNsn com clusters de células neoplásicas, algumas células isoladas e um LFNñsn positivo devido a presença de uma micrometástase detectada no HE.

A factibilidade da técnica foi de $100 \%$. A acurácia do método foi de $83 \%$ quando utilizamos somente o HE, e de 91,6\% quando utilizado HE mais IHQ. Em 11 de 12 pacientes foi possível predizer as características linfonodais regionais pela concordância da análise anatomopatológica entre os linfonodos sentinelas e não sentinelas.

\section{DISCUSSÃO}

O linfonodo sentinela (LFNsn) é definido como o primeiro linfonodo a receber a drenagem linfática de um tumor, ou seja, em neoplasias que as metástases ocorrem principalmente por via linfática, ele provavelmente será o primeiro local de disseminação ${ }^{19,20,27}$. Várias maneiras de localizar o LFNsn foram descritas. As duas principais são através do uso de corantes e de radiofármacos. Vários tipos de corantes foram usados na pesquisa do linfonodo sentinela em câncer gástri-

Tabela 2 - Características anatomopatológicas dos tumores e tipo de gastrectomia.

\begin{tabular}{|c|c|c|c|c|}
\hline Pacientes & Tipo tumoral & Grau histológico & Tipo macroscópico & Gastrectomia \\
\hline \#1 & difuso & III & Tipo 0 I & Total \\
\hline$\# 2$ & difuso & III & Tipo 3 & Total \\
\hline \#3 & difuso & III & Tipo 3 & Total \\
\hline \#4 & difuso & III & Tipo 2 & Subtotal \\
\hline$\# 5$ & intestinal & III & Tipo 2 & Subtotal \\
\hline \#6 & misto & II & Tipo 3 & Subtotal \\
\hline \#7 & intestinal & I & Tipo 0 IIb & Subtotal \\
\hline$\# 8$ & intestinal & II & Tipo 2 & Subtotal \\
\hline$\# 9$ & difuso & III & Tipo 2 & Subtotal \\
\hline \#10 & difuso & III & Tipo 0 III & Subtotal \\
\hline \#11 & difuso & III & Tipo 0 III & Total \\
\hline$\# 12$ & intestinal & II & Tipo 2 & Subtotal \\
\hline
\end{tabular}

Tabela 3 - Especificações dos linfonodos - LFNsn: número de linfonodos sentinela; LFNsn + : linfonodo sentinela positivos; LFNñsn +: linfonodos não sentinelas positivos; LFNsn N1 e N2 linfonodos sentinelas em relação as estações N1 e estações N2, respectivamente, $\mathbf{L F N}$ total : linfonodos dissecados (LFNsn + LFNñsn) por paciente.

\begin{tabular}{|c|c|c|c|c|c|c|c|}
\hline Pacientes & TNM & LFNsn & LFNsn + & LFNñsn + & LFNsn N1 & LFNsn N2 & LFN total \\
\hline \#1 & pT1N0 & 3 & 0 & 0 & 1 & 2 & 21 \\
\hline \#2 & pT3N1 & 6 & 2 & 1 & 4 & 2 & 25 \\
\hline$\# 3$ & pT3N0 & 3 & 0 & 0 & 2 & 1 & 24 \\
\hline \#4 & pT3N1 & 3 & 2 & 5 & 3 & 0 & 12 \\
\hline$\# 5$ & pT2N1 & 3 & 1 & 0 & 2 & 1 & 19 \\
\hline \#6 & pT3N1 & 6 & 0 & 5 & 4 & 2 & 27 \\
\hline$\# 7$ & pT1N0 & 2 & 0 & 0 & 1 & 1 & 10 \\
\hline \#8 & pT2N1 & 2 & 1 & 0 & 2 & 0 & 18 \\
\hline \#9 & pT2N0 & 2 & 0 & 0 & 2 & 0 & 24 \\
\hline \#10 & pT1N1 & 2 & 2 & 3 & 2 & 0 & 10 \\
\hline \#11 & pT1N0 & 3 & 0 & 0 & 2 & 2 & 21 \\
\hline \#12 & pT2N1 & 4 & 1 & 1 & 2 & 2 & 25 \\
\hline
\end{tabular}




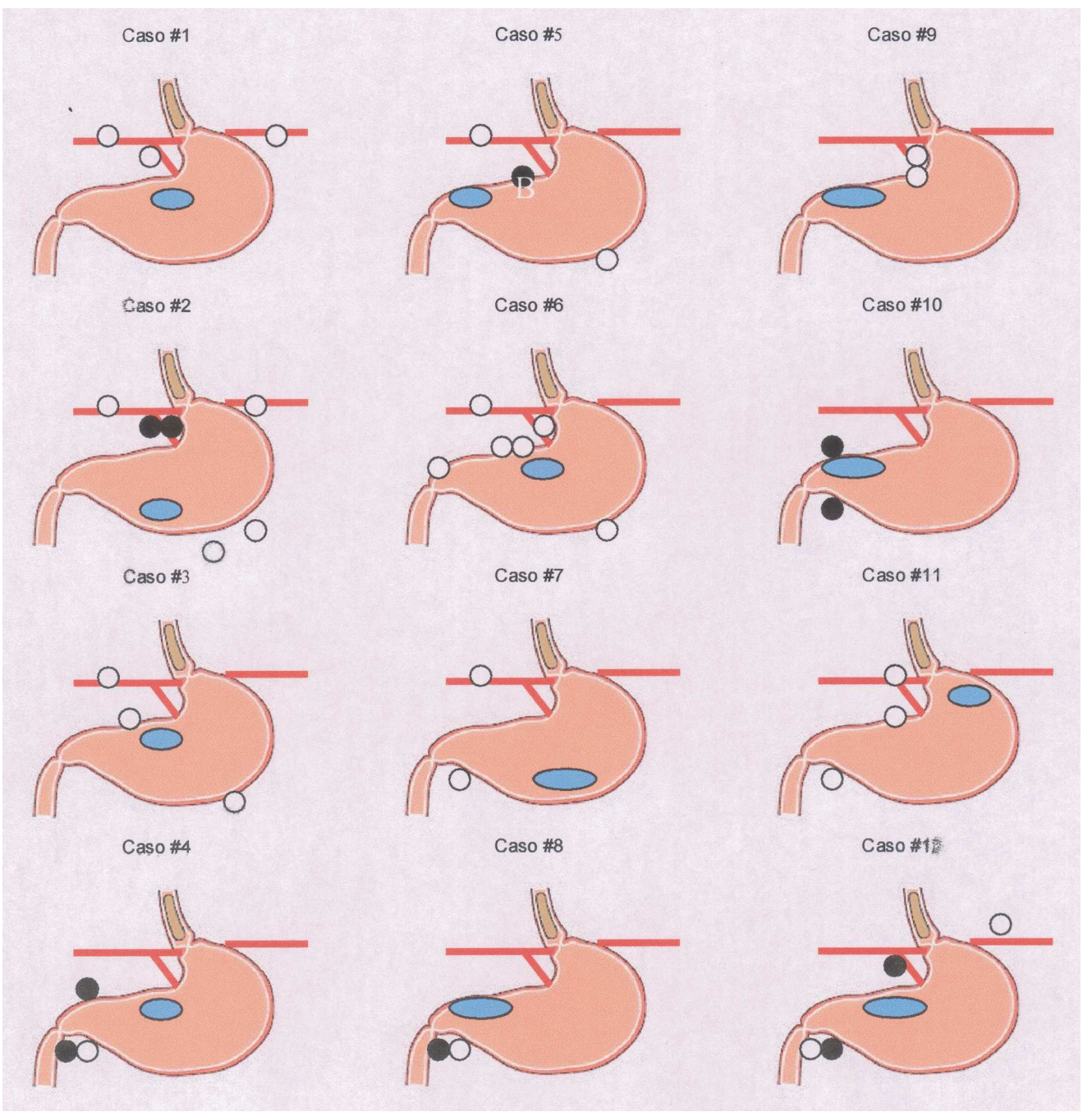

Figura 1 - Esquema gráfico com localização tumoral e respectivos LFNsn. Tumor em cor azul clara, linfonodos sentinelas negativos em branco e linfonodo sentinela positivos em preto.

co, entre eles, índigo carmim, azul patente, azul de Evans, azul de metileno, isosulfan blue, partículas de carbono ativado e o verde de indocianina ${ }^{10}$. No nosso estudo usamos o corante azul patente devido à freqüente utilização do mesmo na pesquisa do LFNsn em câncer de mama e melanoma e também por apresentar um baixo custo comercial, fácil manuseio e baixo potencial alergênico.

Alguns autores utilizaram a associação dos dois métodos, corante e radiofármaco, e obtiveram resultados semelhantes aos que empregaram um método isolado ${ }^{6,9,11}$. No presente estudo foi possível identificar em 100\% (12/12) dos pacientes os LFNsn. Este tipo de corante já demonstrou ser um adequado marcador linfático e comprovamos isto no nosso estudo.

O número médio de LFNsn foi de 3,2 por paciente, número semelhante ao da maioria dos estudos que utilizaram o corante - $-9,13,14$. O emprego exclusivo do corante ou os dois métodos associados demonstrou ser adequado em várias publicações ${ }^{5,6,8,10,11-14,28}$. Neste estudo, inicialmente propusemos a utilização dos dois métodos, radiofármaco e o corante, mas foi constatado que a injeção do radiofármaco não poderia ser feita da mesma forma que o corante. A razão principal foi o extravasamento de pequenos volumes do radiofármaco no ponto de injeção na parede gástrica, ocasionando uma contaminação da cavidade abdominal pelo radiofármaco o que impossibilita o rastreamento dos LFNsn com o gama-probe intraoperatório. Outro detalhe prático é que o radiofármaco deveria ser injetado através de endoscopia digestiva alta algumas horas antes do procedimento para propiciar um esvaziamento dos resíduos radioativos do estômago e isto tornou o método de difícil realização devido a logística de funcionamento dos hospitais que participaram do nosso estudo ${ }^{8,11,13}$.

A visualização dos LFNsn ocorreu de forma rápida e fácil. O tempo determinado de cinco minutos demonstrou ser adequado. Em alguns pacientes o tempo de um a dois minutos foi suficiente para visualizarmos os linfonodos corados. Este tempo está de acordo com a maioria das publicações que utilizaram o corante como marcador ${ }^{5,6,8,9}$. A cor azul do corante é excelente porque contrasta com a cavidade abdominal e seus órgãos, apenas podendo causar confusão com veias muito próximas a LFNsn de pequenas dimensões. Um detalhe técnico que deve ser observado diz respeito à retirada da agulha após injeção do corante, a qual deve ser mantida sob aspiração e seguida de compressão com gaze 
cirúrgica durante alguns segundos, pois pode ocorrer extravasamento para a cavidade peritoneal o que dificultaria a visualização dos LFNsn.

Maruyama et al $^{4}$ apresentaram em 1999 estudos com LFNsn em câncer gástrico. Através de um corante de partículas de carbono ativadas demonstraram trajetos linfáticos e linfonodos sentinelas. Verificaram a existência freqüente de trajetos linfáticos que se originavam na zona peri-tumoral e migravam para linfonodos do segundo nível (skip metastasis). Observaram uma rede complexa de conexões e sugeriram que as células tumorais poderiam, através destes canais, levar metástases não somente para os linfonodos, mas por migração por dentro de alguns linfonodos sem necessariamente ficarem restritas a este linfonodo. Os autores acima concluíram que era muito cedo para utilizar-se o LFNsn para poder limitar a extensão da linfadenectomia regional.

Na nossa casuística foram encontrados oito(66\%) pacientes em que os linfonodos sentinelas também estavam presentes em cadeias extra-perigástricas. Com isso podemos entender o conceito de skip metastases (metástases saltadoras), o qual se baseou em estudos das drenagens linfáticas em que os linfonodos que recebiam a drenagem gástrica se encontravam em linfonodos extra-perigástricos ${ }^{15}$.

Tsolias et al ${ }^{29}$, publicaram em 2000 , seis casos de linfonodo sentinela em câncer gástrico utilizando o corante isosulfan blue encontrando 1,7 LFNsn por paciente. Neste artigo, devido à presença de tumores de outras localizações e ao baixo número de pacientes, não foi possível determinar características específicas do grupo de pacientes estudados, mas verificou-se o adequado uso do corante e, como conclusão, os autores referiram que o mapeamento linfático e a pesquisa do LFNsn são factíveis, e que o linfonodo sentinela reflete as características anatomopatológicas dos linfonodos regionais. Esta representou a primeira publicação com resultados favoráveis ao uso do LFNsn em câncer gástrico, apesar de não estudar exclusivamente este tipo de tumor.

A primeira grande série de LFNsn em câncer gástrico foi publicada em março de $2001^{5}$, em 74 pacientes portadores de tumores T1 e T2 de estômago. O corante utilizado foi o verde de indocianina. Foram encontrados em 73 dos 74 casos o LFNsn (99\% de sucesso), com uma média de 2,6 linfonodos sentinelas por paciente. A sensibilidade do método foi de $90 \%$ e $100 \%$ de especificidade. Neste mesmo ano, duas outras publicações apresentaram resultados satisfatórios com o uso da combinação de corante e radiofármaco. Aikou et $a l^{6}$, apresentaram uma série de 18 casos com identificação em $94 \%$, todos os pacientes com tumores precoces (T1). Foram encontrados três LFNsn em média, variando de zero a seis por paciente. Kitagawa $e a l^{9}$, apresentaram resultados preliminares em vários tipos de tumores como de cólon, esôfago e estômago. Quanto ao câncer gástrico foram pesquisados 16 pacientes com sucesso de $88 \%$ e uma média de 3,5 linfonodos por caso. Verificou-se nesta publicação o primeiro relato da pesquisa do LFNsn com o uso do gama-probe em videolaparoscopia , obtendo o mesmo número de LFNsn por paciente em relação a operação convencional.

Em 1907, Jamieson et al ${ }^{30}$, observaram, em cadáveres, características de migração para linfonodos regionais em tumores gástricos. Estudos da drenagem linfática gástrica revelaram uma variabilidade biológica muito grande, porém verificou-se que o fluxo linfático pode ser avaliado pelo estudo das metástases linfonodais que seguem as cinco principais artérias de irrigação gástrica ${ }^{12}$. Verificamos no nosso estudo o corante passando através de vários linfonodos e, às vezes, pelas estações linfonodais, demonstrando uma complexidade grande no sistema linfático gástrico. Além das variações biológicas que podem demonstrar rotas linfáticas aberrantes, mudanças na drenagem podem ocorrer por obstrução tumoral linfática ${ }^{4}$. Pacientes que apresentam doença ulcerosa gástrica também apresentam pontos de obstrução linfática por conseqüência de processo inflamatório local ${ }^{31}$. Pacientes com doença macroscópica em linfonodos regionais podem ter alterações na migração de marcadores de linfonodos sentinelas (corante e/ou radiofármaco). Outra causa de não migração do corante ou do radiofármaco é a presença de lesão nos linfáticos perigástricos. Isto foi verificado em pacientes excluídos do trabalho onde foram realizadas múltiplas lises de aderências, causando lesões em trajetos linfáticos. Este tipo de problema na migração do corante também foi verificado por Miwa $e t$ $a l^{12}$. Na nossa pesquisa, na paciente \#6, talvez tenha ocorrido uma obstrução linfática por metástases e isto causou uma migração do corante para linfonodos que não apresentavam células tumorais. Esta paciente, em particular, tinha 87 anos, apresentava tumor avançado T3, localizado na pequena curvatura, tipo misto na classificação de Laurén e com três $\mathrm{cm}$ de diâmetro. A migração do corante para linfonodos sem a presença de células tumorais se deve, provavelmente, à obstrução tumoral de canais linfáticos e linfonodos, característica esta já descrita ${ }^{12}$.

Nos pacientes dessa pesquisa foram encontrados LFNsn em estações fora das cadeias perigástricos (N2) em oito casos, o que demonstra que as metástases saltadoras teriam origem neste tipo de visualização, onde o primeiro linfonodo não estaria nas cadeias perigástricas, mas sim em cadeias secundárias (extra-perigástricas).

Estes dados enfatizaram a importância de se ter um método pouco invasivo, mas com poder de estadiar adequadamente os pacientes com câncer gástrico. Em outros tumores, como câncer de mama e melanoma, o LFNsn tornou-se um ótimo método de avaliar metástases linfonodais regionais e prevenir linfadenectomias desnecessárias ${ }^{32,33}$.

Balch CM e Lange JR ${ }^{32}$, descreveram as principais características que os LFNsn deveriam apresentar: 1- proporcionar uma via minimamente invasiva para detectar metástases linfonodais, definindo o grupo de pacientes com linfonodos negativos e poupando-os de linfadenectomias; 2 - Permitir um reconhecimento precoce de metástases comparado com exames clínicos e radiológicos, e com isso proporcionar linfadenectomias com pequeno volume de doença; 3 - Ter um método que promova uma melhor acurácia do estadiamento, porque pode detectar linfonodos sentinelas fora das áreas de drenagens convencionais e melhor avaliação de micrometástases; 4 - Melhorar a acurácia, a interpretação e a comparabilidade entre estudos; 5 - Deve também ser uma poderosa ferramenta para examinarmos a biologia das metástases linfáticas. 
Um grande avanço que o uso do linfonodo sentinela proporcionou foi a possibilidade de melhorar a análise anatomopatológica com aumento de chances em visualizar micrometástases em linfonodos ${ }^{1,2,34}$. Para estes casos não existe ainda uma conduta definida para conduzir o tratamento e nem se sabe qual o comportamento deste tipo de metástases ${ }^{35-37}$. Vários trabalhos correlacionam a presença de micrometástases com o prognóstico, no entanto os resultados são conflitantes $^{1,34,35}$. A avaliação anatomopatológica inicial do LFNsn na nossa pesquisa foi a mesma dos demais linfonodos. No entanto nos casos em que não foram encontrados linfonodos positivos foi realizada análise dos linfonodos sentinelas com múltiplos cortes, realizada coloração com HE e imuno-histoquímicao o que ocorreu em seis pacientes. Nestes pacientes confirmou-se negatividade em cinco casos tanto para HE quanto IHQ. Um apresentou positividade somente na IHQ, revelando uma micrometástase e em outro LFNsn deste paciente, clusters e células neoplásicas isoladas. Após a verificação com a IHQ, foram revistas as lâminas com HE e nelas foi possível observar a mesma micrometástase. A simples revisão pela HE pode ser falha, como já foi citada em várias publicações onde a imuno-histoquímica pode criar um "up-stage" (migração para cima) no estadiamento de pacientes com câncer gástrico em aproximadamente $30 \%$ 35,36.

O LFNsn poderá ser uma ferramenta poderosa para auxiliar procedimentos minimamente invasivos , onde tumo- res precoces com baixo potencial de metástases linfonodais poderiam apenas receber a ressecção do tumor e a pesquisa do LFNsn .

O linfonodo sentinela em câncer gástrico tem se mostrado um método adequado de avaliação de doença linfonodal regional. Esperamos que, assim como no câncer de mama e no melanoma, o linfonodo sentinela possa vir a fazer parte da rotina de estadiamento de tumores gástricos. Em nossa pesquisa conseguimos uma acurácia de 91,6\% $(11 / 12)$ em predizer as características anatomopatológicas dos demais linfonodos regionais. Somente em um paciente não foi possível predizer as características dos linfonodos regionais.

Talvez o grande papel do LFNsn seja em tumores precoces onde as possibilidades de metástases são menores e bem conhecidas. Os pacientes portadores deste tipo de tumor poderão ser poupados de extensas linfadenectomias quando linfonodos sentinelas não evidenciarem metástases, ou apresentaram micrometástases, diminuindo a morbidade e mortalidade que existe hoje nestes procedimentos.

A pesquisa do linfonodo sentinela em câncer gástrico é factível com o uso da tácnica do corante azul patente. $\mathrm{O}$ método mostrou ser promissor como técnica minimamente invasiva para estadiar tumores gástricos, nesta casuística inicial.

\begin{abstract}
Background: The objective was to determine the feasibility of using vital blue dye to map sentinel lymph nodes (SLN) in gastric cancer using a minimally invasive technique. Methods: Sentinel lymph node biopsy was performed on 12 patients with gastric adenocarcinoma that did not have metastatic lymph nodes during clinical staging. Vital blue dye was injected around four quadrants of the tumor, $0.5 \mathrm{ml}$ in each quadrant. The lymph nodes that stained blue 5 minutes after dye injection were classified as SLN. Histopathological analysis of the sentinel lymph nodes was done according to routine evaluation of other lymph nodes, with hematoxylin and eosin $(H E)$ staining. If the SLN was negative for malignancy by means of HE, it was further examined by immunohistochemistry. Results: Sentinel lymph node biopsies in gastric cancer patients were performed from July 2002 to December 2004. Twelve patients were enrolled at the study, being 8 women, with a mean age of 64.5 years (range, 48 to 87). A mean of 3.25 SLNs (range, 2 to 6) were identified in each patient. The feasibility of the procedure was 100\%, and its accuracy was $91.6 \%$. In 11 of 12 patients, it was possible to predict the regional lymph nodes status by concordance of the histopathological analysis of SLN and non-sentinel lymph nodes (nSLN). Conclusion: The sentinel lymph node biopsy in gastric cancer technique has demonstrated to be feasible, with the use of vital blue dye. The procedure is promising as a minimally invasive technique of gastric tumors staging, in our series.
\end{abstract}

Key words: Stomach neoplasms; Lymphatic metastasis; Neoplasm staging; Sentinel lymph node biopsy; Surgery, computer-assisted.

\section{REFERÊNCIAS}

1. Karube T, Ochiai T, Shimada H, Nikaidou T, Hayashi H. Detection of sentinel lymph nodes in gastric cancers based on immunohistochemical analysis of micrometastases. J Surg Oncol. 2004;87(1):32-8.

2. Uenosono Y, Natsugoe S, Ehi K, Arigami T, Hokita S, Aikou T. Detection of sentinel nodes and micrometastases using radioisotope navigation and immunohistochemistry in patients with gastric cancer. Br J Surg. 2005; 92(7):886-9.

3. Osborne MP, Rosenbaum-Smith SM. The historic background of lymphatic mapping. In: Cody HS III, editor. Sentinel lymph node biopsy. London: Martin Dunitz; 2002. p. 3-10.
4. Maruyama K, Sasako M, Kinoshita T, Sano T, Katai H. Can sentinel node biopsy indicate rational extent of lymphadenectomy in gastric cancer surgery? Fundamental and new information on lymph-node dissection. Langenbecks Arch Surg. 1999;384(2):14957.

5. Hiratsuka M, Miyashiro I, Ishikawa O, Furukawa H, Motomura K, Ohigashi H, Kameyama M, Sasaki Y, Kabuto T, Ishiguro S, Imaoka S, Koyama H. Application of sentinel node biopsy to gastric cancer surgery. Surgery. 2001;129(3):335-40.

6. Aikou T, Higashi H, Natsugoe S, Hokita S, Baba M, Tako S. Can sentinel node navigation surgery reduce the extent of lymph node dissection in gastric cancer? Ann Surg Oncol. 2001;8(9 Suppl):90S-93S. 
7. Kitagawa Y, Fujii H, Mukai M, Kubota T, Otani Y, Kitajima M. Radio-guided sentinel node detection for gastric cancer. Br J Surg. 2002;89(5):604-8

8. Kim MC, Kim HH, Jung GJ, Lee JH, Choi SR, Kang DY, Roh MS, Jeong JS. Lymphatic mapping and sentinel node biopsy using $99 \mathrm{mTc}$ tin colloid in gastric cancer. Ann Surg. 2004;239(3):383-7.

9. Kitagawa Y, Ohgami M, Fujii H, Mukai M, Kubota T, Ando N, Watanabe M, Otani Y, Ozawa S, Hasegawa H, Furukawa T, Matsuda J, Kumai K, Ikeda T, Kubo A, Kitajima M. Laparoscopic detection of sentinel lymph nodes in gastrointestinal cancer: a novel and minimally invasive approach. Ann Surg Oncol. 2001;8(9Suppl):86S-89S.

10. Ichikura T, Morita D, Uchida T, Okura E, Majima T, Ogawa T, Mochizuki H. Sentinel node concept in gastric cancer. Word J Surg. 2002;26(3):318-22. Epub 2001 Dec 17.

11. Hayashi H, Ochiai T, Mori M, Karube T, Suzuki T, Gunji Y, Hori S, Akutsu N, Matsubara H, Shimada H. Sentinel lymph node mapping for gastric cancer using a dual procedure with dye and gamma probe guided techniques. J Am Coll Surg. 2003;196(6):68-74.

12. Miwa K, Kinami S, Tanaguchi K, Fushida S, Fujimura T, Nonomura A. Mapping sentinel nodes in patients with earlystage gastric carcinoma. Br J Surg. 2003;90(2):178-82.

13. Yasuda S, Shimada H, Chino O, Tanaka H, Kenmochi T, Takechi M, Nabeshima K, Okamoto Y, , Kijima H, Suzuki Y, Ogoshi K, Tajima T, Makuuchi H. Sentinel lymph node detection with Tc$99 \mathrm{~m}$ tin colloids in patients with esophagogastric cancer. Jpn J Clin Oncol. 2003;33(2):68-72.

14. Isozaki H, Kimura T, Tanaka N, Satoh K, Matsumoto S, Ninomiya M, Ohsaki T, Mori M; Esophagus Gastrointestinal Surgical Treatment Study Group. An assessment of the feasibility of sentinel lymph node-guided surgery for gastric cancer. Gastric Cancer. 2004;7(3):149-53.

15. Kitagawa Y, Fujii H, Mukai M, Kubota T, Ando N, Watanabe M, Ohgami M, Otani Y, Ozawa S, Hasegawa H, Furukawa T, Kumai K, Ikeda T, Nakahara T, Kubo A, Kitajima M. The role of sentinel lymph node in gastrointestinal cancer. Surg Clin North Am. 2000; 80(6): 1799-809.

16. Iriyama K, Asakawa T, Koike H, Nishiwaki H, Suzuki H. Is extensive lymphadenectomy necessary for surgical treatment of intramucosal carcinoma of the stomach? Arch Surg. 1989;124(3):309-11.

17. Tsujitani T, Oka S, Saito H, Kondo A, Ikeguchi M, Maeta M, kaibara N. Less invasive surgery for early gastric cancer based on the low probability of lymph node metastasis. Surgery. 1999;125(2):148-54.

18. Kurihara N, Kubota T, Otani Y, Ohgami M, Kumai K, Sugiura H, Kitajima M. Lymph node metastasis of early gastric cancer with submucosal invasion. Br J Surg. 1998;85(6):835-9.

19. Morton DL, Wen DR, Wong JH, Economou JS, Cagle LA, Storm FK, Foshag LJ, Cochran AJ. Technical details of intraoperative lymphatic mapping for early stage melanoma. Arch Surg. 1992;127(4):393-9.

20. Krag DN, Weaver DL, Alex JC, Fairbank JT. Surgical resection and radiolocalization of the sentinel lymph node in breast cancer using a gamma probe. Surg Oncol. 1993;2(6):335-9; discussion 340.

21. Giuliano AE, Kirgan DM, Guenther JM, Morton DL. Lymphatic mapping and sentinel lymphadenectomy for breast cancer. Ann Surg. 1994;220(3):391-8; discussion 398-401.

22. Bonenkamp JJ, Hermans J, Sasako M, van de Velde CJ, Welvaart K, Songun I, Meyer S, Plukker JT, Van Elk P, Obertop H, Gouma DJ, van Lanschot JJ, Taat CW, de Graaf PW, von Meyenfeldt
MF, Tilanus H; Dutch Gastric Cancer Group. Extended lymphnode dissection for gastric cancer. $\mathrm{N}$ Engl J Med. 1999;340(12):908-14.

23. Siewert JR, Böttcher K, Stein HJ, Roder JD. Relevant prognostic factors in gastric cancer: ten-year results of the German Gastric Cancer Study. Ann Surg. 1998;228(4):449-61.

24. Cuschieri A, Weeden S, Fielding J, Bancewicz J, Craven J, Joypaul $\mathrm{V}$, Sydes M. Patient survival after $\mathrm{D}^{1}$ and $\mathrm{D}^{2}$ resections for gastric cancer: long-term results of the MRC randomized surgical trial. Surgical Co-operative Group. Br J Cancer. 1999;79(9-10):1522-30.

25. Sobin LH, Wittenkind Ch, editors. UICC TNM. Classification of malignant tumors. $6^{\text {th }}$ ed. New York: Wiley-Liss; 2003.

26. Japanese Gastric Cancer Association. Japanese Classification of Gastric Carcinoma. 13th ed. Tokio: Kenehara, 1998.

27. Cabanas RM. An approach for the treatment of penile carcinoma. Cancer. 1977;39(2):465-6.

28. Jamieson JK, Dobson JF. Lectures on the lymphatic system of the stomach. Lancet. 1907;I:1061-6.

29. Tsioulias GJ, Wood TF, Morton DL, Bilchik AJ. Lymphatic mapping and focused analysis of sentinel lymph nodes upstage gastrointestinal neoplasms. Arch Surg. 2000;135(8):926-32.

30. Krag D, Weaver D, Ashikaga T, Moffat F, Klimberg VS, Shriver C, Feldman S, Kusminsky R, Gadd M, Kuhn J, Harlow S, Beitsch P. The sentinel node in breast cancer - multicenter validation study. N Engl J Med. 1998;339(14):941-6.

31. Weinberg J, Greaney EM. Identification of regional lymph nodes by means of a vital staining dye during surgery of gastric cancer. Surg Gynecol Obstet. 1950;90(5):561-7.

32. Balch CM, Lange JR. Lymphatic mapping and sentinel node lymphadenectomy for cancer: an overview. Ann Surg Oncol. 2001;8(9 Suppl):1S-4S.

33. Moriya T, Usami S, Tada H, Kasajima A, Ishida K, Kariya Y, Ohuchi N, Sasano H. Pathological evaluation of sentinel lymph nodes for breast cancer. Asian J Surg. 2004;27(4):256-61.

34. Fukagawa T, Sasako M, Mann GB, Sano T, Katai H, Maruyama K, Nakanishi Y, Shimoda T. Immunohistochemically detected micrometastases of the lymph nodes in patients with gastric carcinoma. Cancer. 2001;92(4):753-60.

35. Yasuda K, Adachi Y, Shiraishi N, Inomata M, Takeuchi H, Kitano S. Prognostic effect of lymph node micrometastasis in patients with histologically node-negative gastric cancer. Ann Surg Oncol. 2002;9(8):771-4.

36. Choi HJ, Kim YK, Kim YH, Kim SS, Hong SH. Occurrence and prognostic implications of micrometastases in lymph nodes from patients with submucosal gastric cancer. Ann Surg Oncol. 2001;9(1):13-9.

37. Nakajo A, Natsugoe S, Ishigami S, Matsumoto M, Nakashima S, Hokita S, Baba M, Takao S, Aikou T. Detection and prediction of micrometastasis in the lymph nodes of patients with pN0 gastric cancer. Ann Surg Oncol. 2001;8(2):158-62.36 - Nakajo A, Natsugoe S, Ishigami S, Matsumoto M, Nakashima S, Hokita S, et al. Detection and prediction of micrometastasis in the lymph nodes of patients with pN0 gastric cancer. Ann Surg Oncol 2001; 8:158-62.

Como citar este artigo:

Boff MF, Schirmer CC, Edelweiss MI, Meure L. Pesquisa do linfonodo sentinela em câncer gástrico com o corante azul patente. Rev Col Bras Cir. [periódico na Internet] 2007 Set-Out;34(5). Disponível em URL: www.scielo.br/rcbc

Endereço para correspondência:

Luise Meurer

E-mail: meurerl@terra.com.br 